\title{
Population-Based Design of Mandibular Plates Based on Bone Quality and Morphology
}

\author{
Habib Bousleiman ${ }^{1, \star}$, Christof Seiler ${ }^{1,2}$, Tateyuki Iizuka ${ }^{3}$, \\ Lutz-Peter Nolte $^{1}$, and Mauricio Reyes ${ }^{1}$ \\ 1 Institute for Surgical Technology and Biomechanics, \\ University of Bern, Stauffacherstrasse 78, 3014 Bern, Switzerland \\ \{habib.bousleiman, mauricio.reyes\}@istb.unibe.ch \\ 2 INRIA, Sophia-Antipolis, France \\ 3 Department of Cranio- Maxillofacial Surgery, University of Bern, \\ Inselspital, 3010 Bern, Switzerland
}

\begin{abstract}
In this paper we present a new population-based implant design methodology, which advances the state-of-the-art approaches by combining shape and bone quality information into the design strategy. The method enhances the mechanical stability of the fixation and reduces the intra-operative in-plane bending which might impede the functionality of the locking mechanism. The method is presented for the case of mandibular locking fixation plates, where the mandibular angle and the bone quality at screw locations are taken into account. Using computational anatomy techniques, the method automatically derives, from a set of computed tomography images, the mandibular angle and the bone thickness and intensity values at the path of every screw. An optimisation strategy is then used to optimise the two parameters of plate angle and screw position. Results for the new design are presented along with a comparison with a commercially available mandibular locking fixation plate. A statistically highly significant improvement was observed. Our experiments allowed us to conclude that an angle of $126^{\circ}$ and a screw separation of $8 \mathrm{~mm}$ is a more suitable design than the standard $120^{\circ}$ and $9 \mathrm{~mm}$.
\end{abstract}

Keywords: Orthopaedic implant design, population-based analysis, computational anatomy, mandibular locking fixation plate.

\section{Introduction}

The human mandible is a complex structure and a site of high incidence of traumatic or pathologic defects. Reconstruction of defects of the mandible using internal fixators is a common procedure in the general and specialised orthopaedic wards. Similar to other sites, mandibular fixation plates are selected from a limited range of manufactured models [1. Therefore the need to intra-operatively adapt the implant to the patient-specific anatomy is almost always present.

\footnotetext{
* Corresponding author.
} 
Recent research presented population-based methods to improve the design of the shape of pre-contoured fixation plates 23. In 2], an alteration of a commercially available proximal tibial implant was proposed based on optimisation of surface distances using level-set segmentation in a statistical shape space. In [3], an articulated model of the implant was used to minimise more clinically relevant metrics, namely the amount of bending and torqueing required to adapt the implant to the anatomy of the patient being operated (as opposed to minimising surface distances). However, neither one of the approaches include bone quality information into the design. Moreover, they both focus on the out-of-plane deformations and do not address the in-plane bending of the plates.

For the specific case of locking mandibular plates, in-plane bending is the most important type of deformation since it affects the shape of the screw holes and locking mechanism. Out-of-plane deformations are less important in this case because mandibular plates are thinner, hence much easier to adapt to the patient anatomy. Therefore, for mandibular plates the angle between the mandibular body and ramus, referred to hereafter as mandibular or plate angle, is of major importance.

In addition to the surface morphology, the mechanical properties of bone are a major criterion in the design of implants 4, especially in structures such as the mandible where the amount of bone is not in large supply. A well designed implant must present reduced risk of screw pullout and higher mechanical stability. Bone thickness and bone mineral density can be inferred from computed tomography (CT) images where bone porosity, bone density, and image intensity values are well correlated [5]6. Moreover, it has been shown that the quality of cortical bone has a considerable positive influence on the stability of the implant [7]. For the particular case of the mandibular reconstruction plate, the AO Foundation (Davos, Switzerland) recommends to flush the implant to the inferior edge of the body and posterior edge of the ramus. These regions are almost entirely composed of cortical bone.

In this paper we present a population-based design methodology of orthopaedic fixators, which advances the state-of-the-art approaches by combining shape and bone quality information into the design strategy. The proposed methodology is presented for the specific case of mandibular locking plates. Of special interest is the in-plane pre-contouring and the bone thickness and intensity values at screw insertion sites. The implant parameters that we optimise are the plate angle and the distance between adjacent screw holes. In addition we present a comparison between the proposed design and a commercially available model.

\section{Methods}

\subsection{Experimental Data}

A population of $43 \mathrm{CT}$ images of the adult mandible (gender: $m=25, f=18$ - age: mean $=56$, st.dev $=11.37$, median $=66$ ) was used in this study. Each image is composed of $99 \times 147 \times 145$ voxels and a voxel spacing of $1.25 \mathrm{~mm}$. 


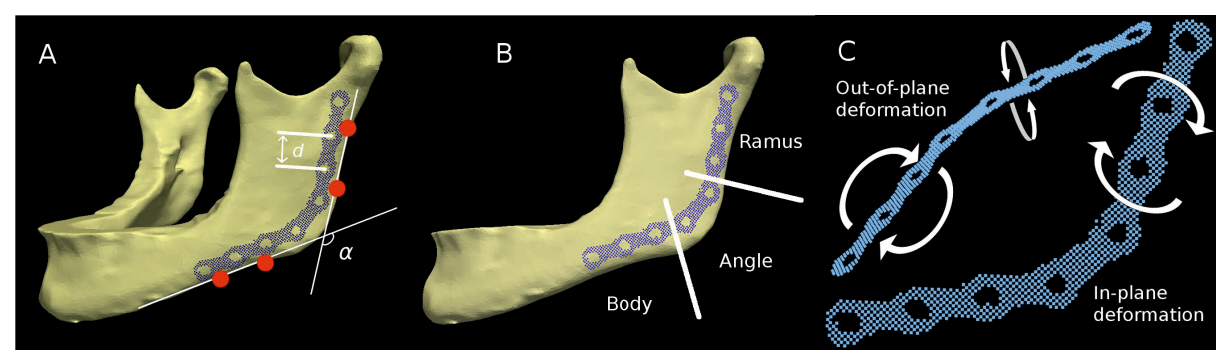

Fig. 1. 3D view of the reference mandible with the standard fixation plate placed in its correct location. (A) Visible landmarks (red circles) used to measure the mandibular angle. $(B)$ Anatomical grouping of the mandible and plate into three regions, namely, ramus, angle, and body of the mandible. $(C)$ Illustration of the in-plane and out-ofplane deformations relative to the plate.

All images were initially rigidly aligned and resampled to the resolution specified above. Non-rigid registration was then applied in order to establish a voxelwise correspondence between every image and a predefined reference. We used the algorithm described in [8] that was initially designed for mandibles and final aim towards implant design. It is a non-linear log-domain demons-based image registration method that uses a hierarchy of locally affine transformations as a regularisation model. The algorithm results in a deformation vector field (DVF) for every image relating voxels in the image to those in the reference via an anatomically meaningful correspondence.

\subsection{Measurement of the Mandibular Angle}

A well-designed angle reduces the need of intra-operative in-plane bending and hence the risk of deforming the locking screw holes. For this reason, we measured the mandibular angle within the population of images in our database. A set of four landmark points was manually placed on the reference mandible. Two landmarks were aligned with the left posterior edge of the ramus and the other two with the left inferior edge of the body of the mandible. The computed DVF was used to propagate the coordinates of the landmarks to all other images in the population. For every instance, the angle between the line connecting the two ramal landmarks and that connecting the landmarks on the mandibular body was automatically measured. Fig. 1A illustrates this process graphically.

\subsection{Measurement of Bone Thickness and Intensity Values}

Similarly, a set of eight landmarks (analogous to the eight screw holes in the standard plate and consistent with AO guidelines, i.e., at least three screws on either side of the fracture) was placed at the screw entry points on the reference image. A corresponding set was also placed on the interior side of the mandible to delineate the paths of the screws. 


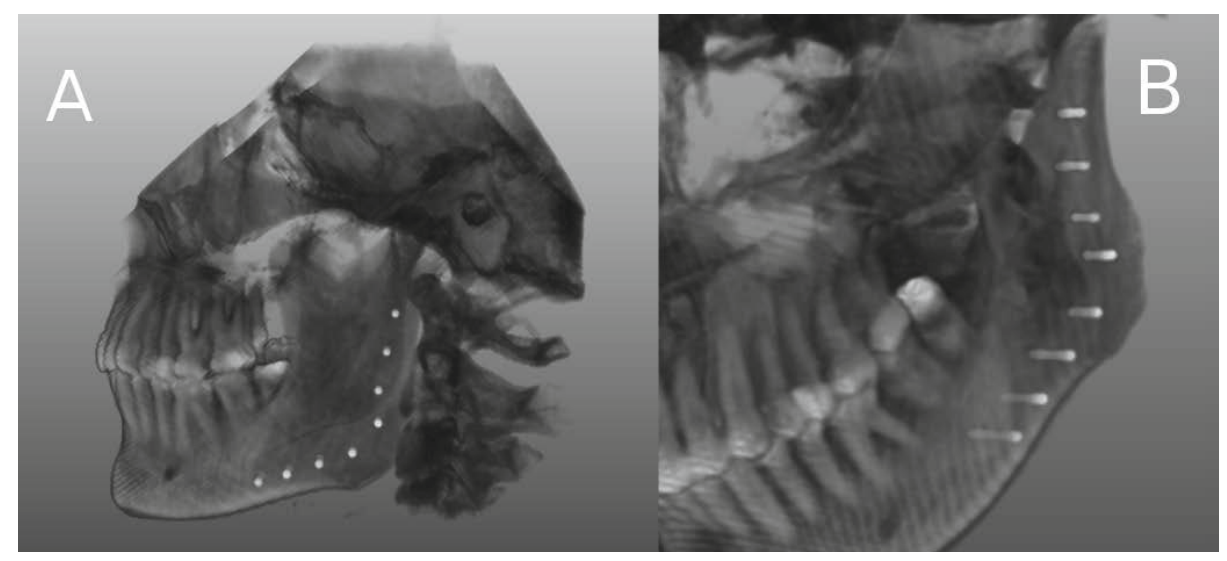

Fig. 2. Volume rendering of the reference image showing the $(A)$ landmarks corresponding to the screw sites of a $120^{\circ}$ plate and $(B)$ the paths of the screws along which the intensity values are sampled

For any particular combination of implant parameters, the landmark configuration is modified accordingly and propagated to all instances of the population using the computed DVF. Geometrical constraints were used in order to ensure that the screw holes remain equidistant, that they are not misaligned, and that the plate angle is not affected by the varying mandibular angle due to the DVF propagation. Additionally, a safety margin of $3 \mathrm{~mm}$ (consistent with the implant dimensions) was set around the screws in order to prevent any parts of the implant from being placed outside the bone.

The Euclidean distance between each pair of corresponding landmarks was computed. This represents the bone thickness at that particular screw insertion site. A $2 \mathrm{~mm}$ tube (diameter of a typical mandibular screws) was used to sample voxels between the same landmarks. The average intensity value was used to represent the cortical bone quality at that location. Fig. 2 shows a $3 \mathrm{D}$ view of the landmark configuration and the sampled volumes.

\subsection{Anatomical Grouping}

Based on recommendations of the AO Foundation and standards of anatomy, the plate and mandible were divided into three anatomically distinct regions, namely, the ramus, the angle, and the body of the mandible. The regions contain three, two, and three screw holes, respectively. Each region was treated separately and considered as a single unit during the optimisation process and the analysis of the results. The two screws of the angle region were used to anchor the plate in place and remain fixed during the optimisation. Therefore, changes in intensity values or bone thickness are not expected for that region. The configuration of the regions is illustrated in Fig. 11B. 


\subsection{Design Process}

The mandibular plate was parametrised using two geometric features, in particular the plate angle $\alpha$ and the distance separating two adjacent screws $d$. The design criteria were the bone thickness and the intensity values per anatomical region $R=\{$ Ramus, Angle, Body $\}$.

For every image in the database, the algorithm scans through the twodimensional search space and for every pair of parameters computes the intermediate energy function

$$
E(\alpha, d)_{i m}=\omega_{\Theta} \sum_{r \in R} \Theta_{r}^{\alpha, d}+\omega_{I} \sum_{r \in R} I_{r}^{\alpha, d} .
$$

$E(\alpha, d)$ is composed of two components, namely, the thickness and the intensity components. Both terms are weighted according to their relative significance. In Eq. (1), $w_{\Theta}$ and $\omega_{I}$ are the respective weighting factors. Both terms are normalised by their respective ranges to a unitless scale with values within $[0,1]$ in order allow linear combination.

The thickness component is the average of the bone thickness measured at all screw sites within one region and can be written as

$$
\Theta_{r \in R}=\frac{1}{N_{r}} \sum_{i=1}^{N_{r}} \tilde{T}_{r, i}
$$

where $N_{r}$ is the number of screws in one particular region and $\tilde{T}$ the normalised local bone thickness.

Similarly, the intensity component is the mean of the intensity values sampled along all screws within one region, or equivalently

$$
I_{r \in R}=\frac{1}{N_{r}} \sum_{i=1}^{N_{r}}\left[\frac{1}{n} \sum_{j=1}^{n} \tilde{H}_{j}\right]_{r, i},
$$

where $n$ is the number of sampled voxels for every screw, and $\tilde{H}$ the normalised individual voxel intensity.

Calculating Eq. (1) for all images results in a vector of length equal to the total number of images $p$ for every combination of $\alpha$ and $d$. The final energy function to be maximised is but the magnitude of the obtained vectors. A traceable extensive search was used, thus eliminating the need for an optimisation strategy such as gradient-based approaches. A formal representation of the total energy function is given as

$$
\begin{aligned}
E(\alpha, d)_{\text {total }} & =\sqrt{\sum_{i m=1}^{p}\left[E(\alpha, d)_{i m}\right]^{2}} \\
& =\sqrt{\sum_{i m=1}^{p}\left[\omega_{\Theta} \sum_{r \in R} \Theta_{r}^{\alpha, d}+\omega_{I} \sum_{r \in R} I_{r}^{\alpha, d}\right]^{2} .}
\end{aligned}
$$




\section{Results}

The first step was to measure the mandibular angle in the population using the approach described above. The mean mandibular angle was found to be $127.30^{\circ}$ with a standard deviation of $6.66^{\circ}$ and a median of $126.56^{\circ}$. These findings were used to initialise and set the domain of the optimisation along the angle parameter. This was chosen to be two standard deviations around the mean mandibular angle of the population with unit steps. The distance between the screws was limited by the screw hole geometry $(6 \mathrm{~mm})$ and an arbitrarily larger value $(16 \mathrm{~mm})$ with unit steps. Different weighting combinations for the energy function were tested. Stable and optimal results were obtained for $\omega_{\Theta} \geq 0.5$ (i.e., $\omega_{I} \leq 0.5$ ). These combinations are consistent with our design strategy to assign higher importance to the available thickness of the bone. We applied the optimisation algorithm on the whole population. Eq. (4) reached its maximum value at $\alpha=126^{\circ}$ and $d=8 \mathrm{~mm}$. The energy function is plotted in Fig. 3 against the $2 \mathrm{D}$ space of design parameters.

In order to evaluate the new design, we generated an implant configuration with the obtained parameters and compared it with that generated using the parameters of the standard design (low-profile MODUS ${ }^{\circledR}$ TriLock ${ }^{\circledR}$ 2.0/2.3/2.5, Medartis AG, Basel, Switzerland. $\alpha=120^{\circ}$ and $d=9 \mathrm{~mm}$ ). We applied the same method in both cases and measured the resulting distribution of bone thickness along the screw insertion paths. We carried out two-tailed t-tests to calculate the statistical significance of the differences in the obtained results using a significance level of 0.05 . The results are shown in Fig. 4 with the statistical significance

\section{Optimisation of the Energy Function}

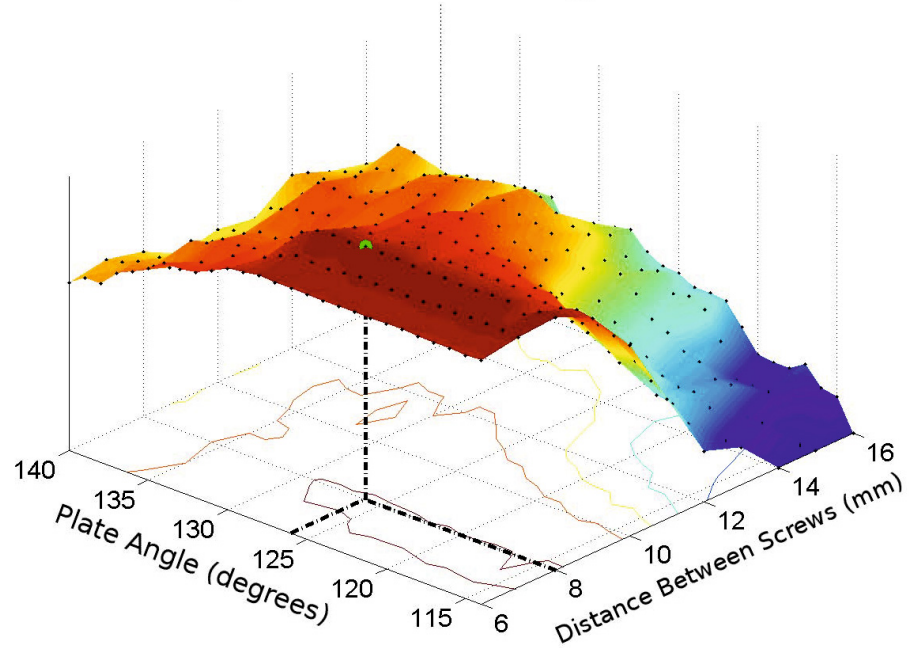

Fig. 3. Surface plot of the energy function against the 2-dimensional optimisation space spanned by the design parameters $\alpha$ and $d$. The green circle is the maximum value. 


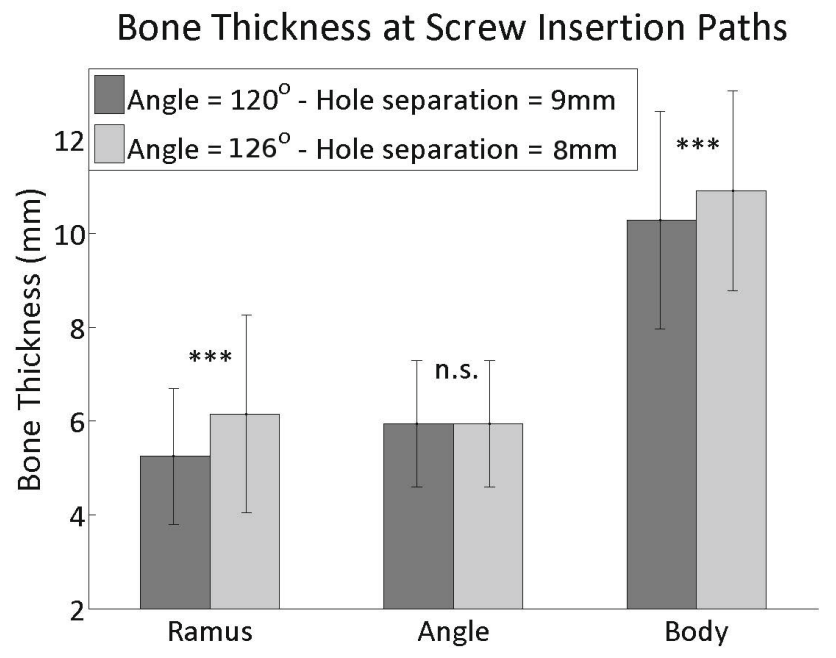

Fig. 4. Comparison of the distribution of bone thickness at the screw insertion paths for the commercially available plate and the proposed new design. The graph shows the results for the three anatomical regions. The statistical significance of the difference between the means of the populations is indicated on the graph (n.s.: not significant, $* * *: p<0.001)$.

marked with asterisks. A net increase in the sampled intensity values of $7.87 \%$ and $23.19 \%$ was also observed for the ramus and body regions, respectively.

\section{Discussion}

In this paper we presented a new population-based orthopaedic implant design methodology that combines shape and bone quality information. The method was presented for the case of mandibular internal reconstruction plates. Of interest to the design were two parameters, namely the angle of the plate and the distance separating two consecutive screw holes. Using computational anatomy techniques, the mandibular angle and the bone thickness and intensity values at screw paths were measured, allowing us to formulate an optimisation strategy to minimise in-plane plate bending and maximise bone quality in the volumes that will be occupied by the screws, which is to a large extent responsible for the success of the fixation. We presented an evaluation of the proposed design by means of a comparison with a commercially available fixator.

The mandibular angle was measured in the population of available CT images. The difference between the measured angle and that of the standard plate indicates that the latter is not optimal and has room for improvement. The proposed and the standard designs were compared and the bone thickness and intensity values at screw locations were recorded. A statistically highly significant increase was measured for the new design in both the ramus and the body 
regions of the mandible. As expected, and since the screws of the mandibular angle were fixed, no change was observed.

The method presented herein can be extended to be applied on other implant types and for various anatomical sites. The length of the implant is not as important as its shape since in practice a plate longer than needed is intra-operatively cut to size. Therefore the length of the fixator was excluded from our analysis. The overall length of the plate will increase with increasing distance between the screw holes. Therefore, the respective effects will be correlated and redundant. We are aware that the length of the fixation might have a direct effect on the mechanical properties of the reconstruction and the force distribution over the locking mechanism. However we have plans to examine this topic in a study involving mechanical and finite element analysis that are outside the scope of this paper.

Acknowledgments. This work was carried out within the frame of the National Centre of Competence in Research, Computer-Aided and Image-Guided Medical Interventions (NCCR Co-Me), supported by the funds of the Swiss National Science Foundation (SNSF).

\section{References}

1. Nagamune, K., Kokubo, Y., Baba, H.: Computer-assisted designing system for fixation plate. In: IEEE International Conference on Fuzzy Systems - FUZZ IEEE, Jeju Island, Korea, pp. 975-980 (2009)

2. Kozic, N., Weber, S., Büchler, P., Lutz, C., Reimers, N., González, M., Reyes, M.: Optimisation of orthopaedic implant design using statistical shape space analysis based on level sets. Medical Image Analysis 14, 265-275 (2010)

3. Bou-Sleiman, H., Ritacco, L.E., Nolte, L.-P., Reyes, M.: Minimization of IntraOperative Shaping of Orthopaedic Fixation Plates: A Population-Based Design. In: Fichtinger, G., Martel, A., Peters, T. (eds.) MICCAI 2011, Part II. LNCS, vol. 6892, pp. 409-416. Springer, Heidelberg (2011)

4. Schiuma, D., Brianza, S., Tami, A.E.: Development of a novel method for surgical implant design optimization through noninvasive assessment of local bone properties. Medical Engineering \& Physics 33, 256-262 (2011)

5. Merheb, J., Van Assche, N., Coucke, W., Jacobs, R., Naert, I., Quirynen, M.: Relationship between cortical bone thickness or computerized tomography-derived bone density values and implant stability. Clinical Oral Implants Research 21, 612-617 (2010)

6. Zhang, J., Yan, C.-H., Chui, C.-K., Ong, S.H.: Accurate measurement of bone mineral density using clinical CT imaging with single energy beam spectral intensity correction. IEEE Transactions on Medical Imaging 29, 1382-1389 (2010)

7. Hong, J., Lim, Y.-J., Park, S.-O.: Quantitative biomechanical analysis of the influence of the cortical bone and implant length on primary stability. Clinical Oral Implants Research, 1-5 (2011)

8. Seiler, C., Pennec, X., Reyes, M.: Geometry-Aware Multiscale Image Registration via OBBTree-Based Polyaffine Log-Demons. In: Fichtinger, G., Martel, A., Peters, T. (eds.) MICCAI 2011, Part II. LNCS, vol. 6892, pp. 631-638. Springer, Heidelberg (2011) 